\title{
Acoustic microscopy in the food industry
}

\author{
Nicholas Watson ${ }^{1}$, Malcolm Povey ${ }^{1}$, Edith Corona $^{2}$ and Nicholas Parker ${ }^{3}$ \\ ${ }^{1}$ Department of Food Science and Nutrition, University of Leeds, Leeds LS2 9JT, UK. \\ ${ }^{2}$ Grupo de Análisis y Simulación de Procesos Agroalimentarios, Departamento Tecnología de \\ Alimentos, Universidad Politécnica de Valencia, Camí de Vera s/n, E46022, Valencia, Spain. \\ ${ }^{3}$ School of Mathematics and Statistics, Newcastle University, Newcastle NE17RU, UK. \\ E-mail: n.j.watson@leeds.ac.uk
}

\begin{abstract}
Acoustic microscopy has been used for many years to image and measure the elastic properties of materials across a wide range of scientific disciplines. However the application of this technique in the food industry is scarce. In this paper we outline the operation of an reflection-mode acoustic microscope and discuss some of the issues relevant to its operation in the food sector. We then present two relevant case studies in which we employ acoustic microscopy to analyse potato cells and the fat structure in Iberian ham and chorizo.
\end{abstract}

\section{Introduction}

Acoustic microscopy is the study of materials on a microscopic scale using focused sound waves, as first demonstrated by Lemons and Quate [1] in 1974 to study biological tissue. Benefits of acoustic microscopy include its potential to perform sub-surface imaging in optically-opaque materials, it does not require dyeing of samples and the image contrast is controlled by mechanical properties. Often the main challenge is in interpreting the complex data to give meaningful results. A scanning acoustic microscope (SAM) works by using positioning system to move a focused acoustic transducer relative to a sample. Reflection-mode SAMs are the most common microscope use a single transducer to both transmit and receive the sound waves. Although acoustic microscopes exist with different transducer arrangements, reflection-mode SAMs are the most popular due their relative simplicity and versatility, and will be considered in this paper. More detailed information on the theory, design and wider applications of acoustic microscopes can be found in several books and review articles $[2,3,4]$.

Acoustic microscopes have been used extensively to study electrical components, composite material and biological systems, but their use in the food sector has been limited [5]. This paper will discuss the main features and components of a reflection-mode acoustic microscope and present two ongoing case studies which highlight the benefits and challenges of using an acoustic microscope in the food sector.

\section{Operational considerations}

\subsection{Acoustic transducer}

The most important component in the SAM is the focused acoustic transducer. The transducer generates the sound waves which interacts with the sample and receives the acoustic information which is used to provide images and measurements of the sample. The possibility of the generation of surface waves when the sound interacts with the surface will be neglected here. The important selection criterion for the acoustic transducer is the operating frequency. This frequency governs the imaging resolution of the transducer as well as the propagation depth, as shown in Figure 1 for a range of frequencies generally used in acoustic microscopy. A trade off exists between resolution and 
propagation depth so the operator must select the most suitable frequency for the sample they are using and the data they wish to require. Shown is a representation of the propagation distance through a homogeneous fluid; the presence of a sample will further modify the propagation distance depending on, for example, the sound transmission into the sample and the sample's acoustic attenuation. A more detailed description of the operation of our SAM can be found in [6].

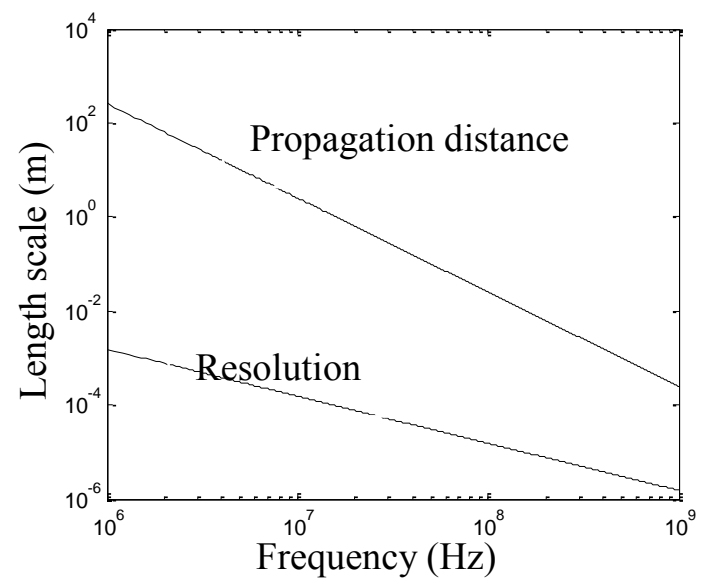

Figure 1: Propagation distance and resolution as a function of frequency, adapted from [7]. This assumes sound propagation in pure water at $30{ }^{\circ} \mathrm{C}$.

\subsection{Scan type}

At each scan point, the returning sound waves received by the transducer generate a voltage which varies with time-of-flight. This voltage "trace" is the basis for constructing images of the sample. Depending on the imaging requirements by a user, the SAM can be operated in A-scan, B-scan and Cscan mode. An A-scan is a time-of-flight measurement performed at a single lateral position, a B-scan consists of multiple time-of-flight measurements performed laterally across the sample (X or Y) and a C-scan consists of multiple measurements across an area (X and $\mathrm{Y}$ ) taken at constant time-of-flight. For surface imaging, C-scans are generally used. However, C-scans are the most time and data intensive and for certain requirements, e.g. monitoring properties which change rapidly with time, faster A-scans and B-scans may be preferable.

\subsection{Sample preparation}

An important part of acoustic microscopy which is little discussed is sample preparation. The top surface of the sample must be as flat and horizontal as possible as any deviations will cause large angle reflections and reduced received signals. Furthermore, for high resolution imaging, for which the depth of focus may be of the order of tens microns, an uneven surface may lead to only certain sections being in focus. To obtain flat samples equipment such as a microtome can be used. Often biological samples are fixed and mounted in wax before slicing and imaging. These techniques can be used for acoustic microscopy work although care must be taken when acquiring the elastic properties of fixed cells as these processes can change the cell's properties. Furthermore, the fixing and mounting method may directly affect the sound propagation in the sample.

Acoustic microscopes require an immersion fluid to couple the acoustic wave between the transducer and sample. Air is unsuitable due to high attenuation and sensitivity to fluctuations. Water is commonly used due to its excellent transmission properties and well-calibrated acoustic behaviour. Food samples which are water soluble can be accommodated by either using another coupling fluid such as oil, or to cover the sample with a thin layer of microfilm which can remain essentially transparent to the sound waves.

\section{Potatoes}


Potatoes are one of the most important foods today yet $20 \%$ of crops can be compromised due to problems such as bruising [8]. Currently there is a limited understanding of why certain potatoes bruise more easily than others. Acoustics offer the potential to study the cellular properties of potatoes and we will present some preliminary work into this area.

A

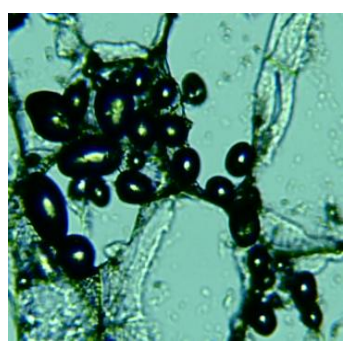

$(500 \times 500 \mu \mathrm{m})$
B

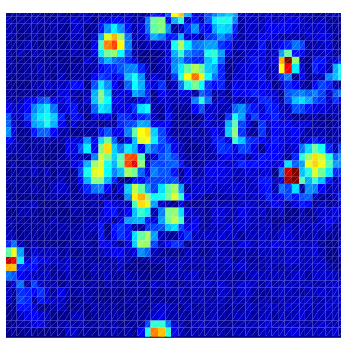

$(500 \times 500 \mu \mathrm{m})$
$\mathrm{C}$

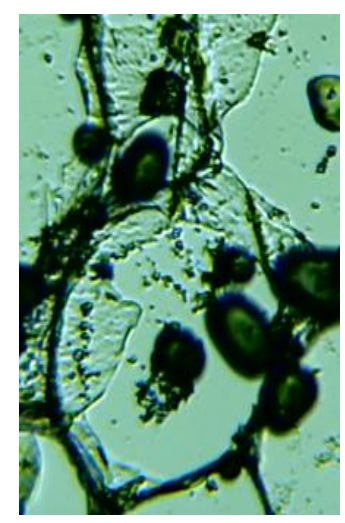

$(150 \times 200 \mu \mathrm{m})$
$\mathrm{D}$

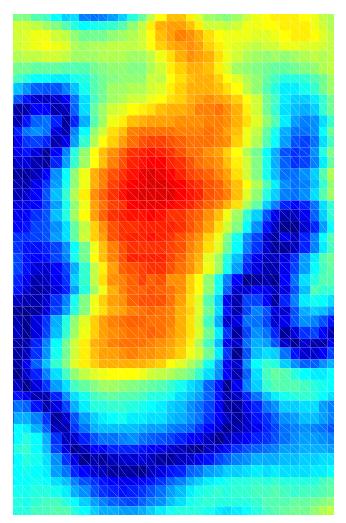

$(150 \times 200 \mu \mathrm{m})$

Figure 2: Optical $(\mathrm{A}, \mathrm{C})$ and acoustic $(\mathrm{B}, \mathrm{D})$ images of potato cells.

A SAM developed in our research group was used to image potato cells. A $100 \mathrm{MHz}$ focused transducer with a $6 \mathrm{~mm}$ focal distance generates a lateral imaging resolution of approximately $20 \mathrm{um}$ [6]. Optical images were obtained with a Bresser LCD microscope. The samples were prepared by fixing, wax mounting and slicing using a Bright M3500 microtome.

From Figure 2 it can be seen that the key components of potatoes are the cell walls and starch granules. The optical images (A and C) show both the starch and cell walls. Using the SAM (B and D) it was possible to image these components separately, depending on the $\mathrm{C}$-scan slice chosen. The acoustic images are less clear than the optical counterparts due to the large difference in lateral resolution between the two methods (for the SAM the lateral resolution is around 20 microns, whereas for the optical microscope it is of the order of a micron). In the acoustic images, maximal signal (red regions) arises from the tops of the cells and starch granules, where the surface is locally horizontal and leads to maximal signal returning to the transducer.

\section{Fat in Iberian products}

Pigs can be reared using the intensive method (Cebo) or more natural method (Montanera). The method of rearing can have an impact on the sensory qualities of the fat in the pigs and methods are required to measure these, for example through their mechanical signatures. In this work we used our SAM to study samples of ham and chorizo. Penetration of sound into the samples was highly restricted at $100 \mathrm{MHz}$ (as used in the previous section) due to viscous and scattering effects. Instead, here, we employ a $10 \mathrm{MHz}$ transducer with a lateral resolution of approximately 300 microns.

The two meat samples are shown in Figure 3 (A and B), with the corresponding acoustic B-scans shown in $\mathrm{C}$ and $\mathrm{D}$. In the $\mathrm{B}$-scans, the $\mathrm{x}$-axis displays scan position and the $\mathrm{y}$-axis displays the timeof-flight. The colorscale reflects the magnitude of the reflected signal, with white/black correspond to zero/maximum signal, respectively. The first reflections received $(\sim 7.2 \mu \mathrm{s})$ corresponds to the top surface of the samples. The dashed lines represent the position of the glass slide and signals above this line are reflections and echoes emanating from the slide itself. Thus the region of sample is the intervening region in the plot between the first received signal and the dashed lines. 
A

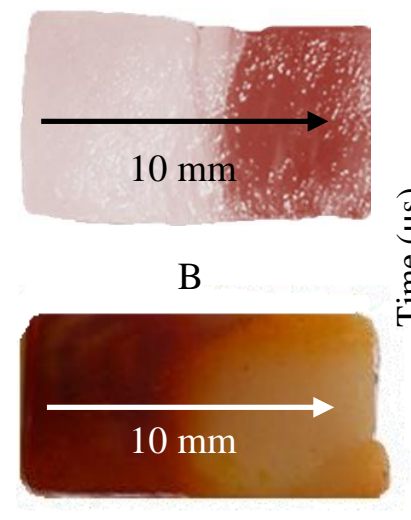

$\mathrm{C}$

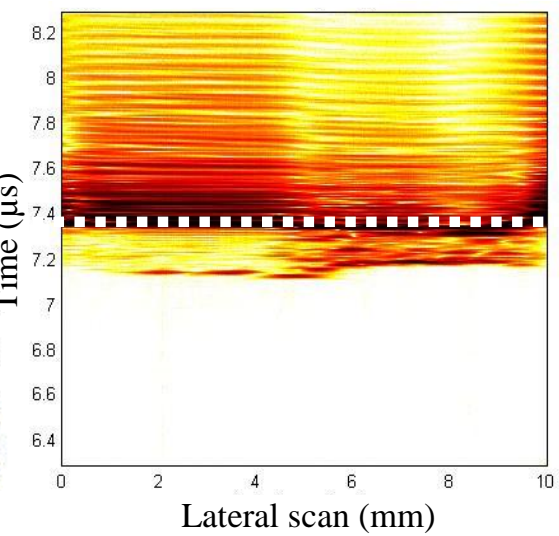

$\mathrm{D}$

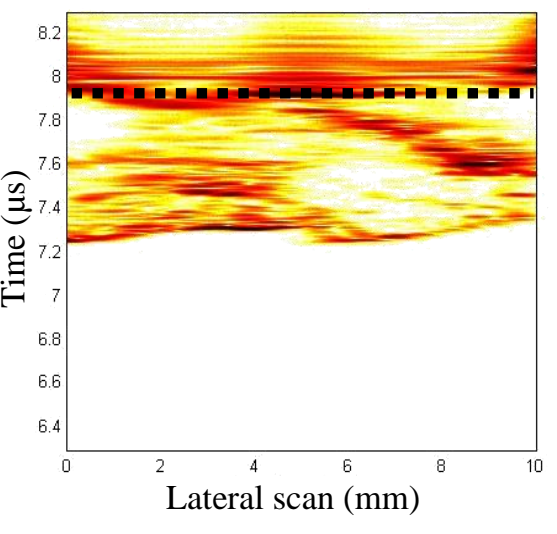

Figure 3: A: Ham sample, B: chorizo sample, C: B-scan of ham, D: B-scan of chorizo.

The ham sample (A) has two distinct regions of fat and muscle. Consistent with this we see a crossover in the B-scan image (C), from a generally light region (left, fat) to a generally dark region (right, muscle). There is a significant difference in acoustic contrast between these two tissue types. Across the whole fluid-sample interface, a large amplitude signal arises due to the large acoustic impedance difference across the interface. Reflections from the subsurface region are caused by backscattering from tissue inhomogeneities, e.g. cell walls, nuclei and proteins. The different structure of fat and muscle tissue leads to very different levels of back-scatter between the tissue types, with the muscle given the larger reflected signals. Within each tissue type, the back-scatter is relatively uniform.

For the chorizo sample (D) the back-scattering from within the sample indicates the very different structure to the ham sample. Here, the sub-surface reflections are inhomogeneous, consistent with the the characteristic presence of globules of fat within the chorizo. At least three fat globules are visible within the sample (light/white regions). These early results suggest that acoustic microscopy has great potential in the study and characterisation of fat/muscle foodtypes.

\section{Conclusion}

In this paper we have briefly described reflection-mode acoustic microscopy and the issues faced by the operator. Our two case studies demonstrate the potential for applying acoustic microscopy within food science and the food industry.

\section{Acknowledgements}

The Authors would like to thank Regiane Scharf for the preparation of the potato samples and the BBSRC for funding (BBB/FOF/302).

\section{References}

[1] Lemons R A and Quate C F 1974 Appl. Phys. Lett. 24 163-165

[2] Yu Z and Boseck S 1995 Rev. Mod. Phys. 67863

[3] Gr Maev R 2008 Acoustic microscopy fundamentals and applications (New Jersey: Wiley$\mathrm{VCH})$

[4] Briggs G A D and Kolosov O 2010 Acoustic microscopy (New York: Oxford Science)

[5] Kaleb M Allan-Wojm P and Shea Miller S 1995 Trends in Food Science \& Technology 6 177186.

[6] N G Parker P V Nelson and M JW Povey 2010 Meas. Sci. Technol. 21

[7] WHITAKER, J. R. \& LEE, C. Y. 1995. Enzymatic Browning and Its Prevention 600 2-7 